\title{
Configurational Temperature Verification of Monte Carlo Simulations
}

\author{
B. D. Butler \\ Physical and Chemical Properties Division \\ National Institute of Standards and Technology* \\ Boulder, CO 80303, USA \\ Gary Ayton, Owen G. Jepps, and Denis J. Evans \\ Research School of Chemistry \\ Australian National University \\ Canberra, ACT 0200, Australia
}

A new diagnostic that is useful for checking the algorithmic correctness of Monte Carlo computer programs is presented. The check is made by comparing the Boltzmann temperature, which is input to the program and used to accept or reject moves, with a configurational temperature $k_{B} T_{\text {config }}=\left|\nabla_{\mathbf{q}} \Phi\right|^{2} / \nabla_{\mathbf{q}}^{2} \Phi$. Here, $\Phi$ is the potential energy of the system and $\nabla_{q}$ represents the dimensionless gradient operator with respect to the particle positions q. We show, using a simulation of Lennard-Jones particles, that the configurational temperature rapidly and accurately tracks changes made to the input temperature even when the system is not in global thermodynamic equilibrium. Coding and/or algorithmic errors can be detected by checking that the input temperature and $T_{\text {config }}$ agree. The effects of system size and continuity of $\Phi$ and its first derivative on $T_{\text {config }}$ are also discussed.

*Contribution of the National Institute of Standards and Technology, not subject to copyright in the U.S.A. 


\section{INTRODUCTION}

When we perform molecular dynamics (MD) computer simulations, the constants of the motion provide useful checks on algorithm validity. While the conservation of momentum and energy do not guarantee the validity of a particular computer program, the vast majority of random programming and algorithmic errors violate these conservation laws and so can easily be detected. Monte Carlo (MC) computer simulations, however, do not conserve momentum or energy, so there are few checks available for algorithm validation. In isothermal-isobaric (NPT) MC, the average output pressure $\mathrm{P}$, computed from the virial, can be compared to the specified input value. Likewise, in grand canonical $(\mu \mathrm{VT}) \mathrm{MC}$, particle insertion methods can be used to compute the chemical potential $\mu$. However, for canonical (NVT) MC, there is no obvious corresponding check on the temperature T. This is because there has been no method of computing the temperature solely from configurational information. There are, therefore, few objective criteria for algorithm validation in the canonical ensemble. This can be particularly troublesome when a new system is being studied and the thermodynamic properties at particular state points cannot be compared to published values.

In this paper, we derive an expression for the temperature, based entirely on configurational information, from fundamental thermodynamic principles. This configurational temperature can be compared to the input temperature in $\mathrm{MC}$ simulations to check the thermodynamic consistency of the computer program.

\section{MATHEMATICAL FORMALISM}

In this section, we derive an expression for the temperature based entirely on the configurations of particles in a simulation and knowledge of the interaction potential. The derivation is analogous to one by $\operatorname{Rugh}^{1}$ for the normal temperature ${ }^{2}$ except that 
here, only the configurational part of the energy in the system (not the kinetic) is used in the expression. From elementary thermodynamics, we have

$$
d E=T d S-p d V \Rightarrow \frac{1}{T}=\left.\frac{\partial S}{\partial E}\right|_{V}
$$

implying that a calculation of the change in the entropy, brought about by an isochoric energy change, will yield the temperature. We denote the phase space vector by $\Gamma \equiv\left(\mathbf{q}_{1}, \ldots, \mathbf{q}_{N}, \mathbf{p}_{1}, \ldots, \mathbf{p}_{N}\right)$ where $\mathbf{q}_{N}$ is the position coordinate of particle $N$ and $\mathbf{p}_{N}$ is its momentum. We define a microcanonical ensemble (NVE) for a given Hamiltonian $H(\Gamma)$ by the set of phase points, denoted $\mu C(E)$, whose energies lie between $E$ and $E+\delta E$, where $|\delta E|<<E$, with Boltzmann's equal a priori probability assumption. The entropy of this ensemble is related to its phase space volume via the relation,

$$
S(E)=k_{B} \ln \left(V_{\Gamma}(E, N, V)\right)=k_{B} \ln \int_{\mu C(E)} d \Gamma
$$

Suppose we displace the phase points $\Gamma$ of our NVE ensemble to the points $\Gamma^{\prime}$ by the transformation

$$
\Gamma^{\prime}(\Gamma)=\Gamma+\frac{\nabla_{\mathrm{q}} H(\boldsymbol{\Gamma})}{\nabla_{\mathrm{q}} H(\boldsymbol{\Gamma}) \cdot \nabla_{\mathrm{q}} H(\boldsymbol{\Gamma})} \Delta E
$$

where $\Delta E$ represents a change in energy of the system and $\nabla_{\mathbf{q}}=\partial / \partial \mathbf{q}_{1}, \ldots, \partial / \partial \mathbf{q}_{N}$ is the configurational gradient of the phase space. That is, we move from one hypersurface to another along a vector

$$
\mathbf{n}(\Gamma)=\frac{\nabla_{\mathbf{q}} H(\Gamma)}{\nabla_{\mathbf{q}} H(\Gamma) \cdot \nabla_{\mathbf{q}} H(\Gamma)}
$$

where only the configurational components of the Hamiltonian are considered. To first 
order in $\Delta E$, this displacement causes a constant change in energy, independent of the initial phase space vector $\Gamma \in \mu C(E)$. Thus, the set of displaced phase points have energy, $E+\Delta E+\mathrm{O}(\Delta E)^{2}$. For our phase space displacement, the Jacobian can be evaluated as

$$
J(\Gamma)=\left|\frac{\partial \Gamma^{\prime}(\Gamma)}{\partial \Gamma}\right|=1+\nabla_{\mathbf{q}} \cdot \mathbf{n}(\Gamma) \Delta E,
$$

where $\nabla_{\Gamma} \equiv \partial / \partial \mathbf{q}_{1}, \ldots, \partial / \partial \mathbf{q}_{N}, \partial / \partial \mathbf{p}_{1}, \ldots, \partial / \partial \mathbf{p}_{N}$ is the phase space gradient operator. The entropy of the $\mathrm{NV}(\mathrm{E}+\Delta \mathrm{E})$ microcanonical ensemble is

$$
S(E+\Delta E)=k_{B} \ln \int_{\mu C(E+\Delta E)} d \boldsymbol{\Gamma}=k_{B} \ln \int_{\mu C(E)} J(\boldsymbol{\Gamma}) d \boldsymbol{\Gamma}
$$

Combining (1), (5), and (6), the temperature $T$ is

$$
\left.\frac{1}{T} \equiv \frac{\partial S}{\partial E}\right|_{V}=k_{B}\left\langle\boldsymbol{\nabla}_{\mathbf{q}} \cdot \mathbf{n}(\boldsymbol{\Gamma})\right\rangle_{E}
$$

Substituting for $\mathbf{n}(\boldsymbol{\Gamma})$ gives

$$
\frac{1}{k_{B} T_{\text {config }}}=\left\langle\boldsymbol{\nabla}_{\mathbf{q}} \cdot\left(\frac{\boldsymbol{\nabla}_{\mathbf{q}} \Phi}{\left|\boldsymbol{\nabla}_{\mathbf{q}} \Phi\right|^{2}}\right)\right\rangle=\left\langle\frac{\boldsymbol{\nabla}_{\mathbf{q}}^{2} \Phi}{\left|\boldsymbol{\nabla}_{\mathbf{q}} \Phi\right|^{2}}\right\rangle+O\left(\frac{1}{N}\right) .
$$

By the equivalence of ensembles, for sufficiently large $N$, (8) will be valid in the canonical ensemble and other ensembles.

We now consider the application of (8) to Monte-Carlo simulations. For sufficiently large $N$, we can estimate the configurational temperature from a single configurational snap-shot without employing ensemble averaging. If the particles interact via a pairwize additive potential 


$$
\Phi=\sum_{i=1}^{N} \sum_{j>i} u_{i j}
$$

the configurational temperature (to leading order in $N$ ) is

$$
k_{B} T_{\text {config }}=\frac{\sum_{i=1}^{N} F_{i}^{2}}{-\sum_{i=1}^{N} \sum_{j \neq i} \nabla_{\mathbf{r}_{i j}} \cdot \mathbf{F}_{i j}},
$$

where,

$$
\mathbf{F}_{i}=\sum_{j \neq i} \mathbf{F}_{i j}=-\sum_{j \neq i} \frac{\partial u_{i j}}{\partial \mathbf{r}_{i j}}
$$

is the total force acting on particle $i$ and $\mathbf{r}_{i j}$ is the vector separation between particles $i$ and

$j$. Unlike the input temperature, there will be a statistical uncertainty in $T_{\text {config }}$ from (10), but this can be improved by averaging the results from several snap-shots taken over times where the configurations are uncorrelated or by increasing the number of particles in the simulation.

\section{RESULTS}

\subsection{Equilibration of $T_{\text {config }}$}

Our derivation of the configurational temperature $T_{\text {config }}$ is based on equilibrium statistical mechanics. The derivation assumes that at constant energy, phase space is uniformly occupied. It is not obvious whether the configurational temperature so derived has any meaning for thermodynamically metastable or unstable states where the equal a priori probability assumption has broken down. To examine this we performed Monte-Carlo simulations of a two-dimensional system that is quenched deep inside the gas/solid 
coexistence region. For a long time after the quench, as decomposition proceeds, the system is not in thermodynamic equilibrium.

The system consisted of a large number, $N=50000$, of particles interacting through the Lennard-Jones potential

$$
u_{i j}=4\left(\frac{1}{r_{i j}^{12}}-\frac{1}{r_{i j}^{6}}\right)
$$

The Monte-Carlo simulation was performed in the canonical ensemble, at a density $\rho=N / A=0.35$, with the potential truncated at $r_{i j}=6$. The system was equilibrated at $T=1$ (well above the critical temperature) and then quenched deep inside the coexistence region to $T=0.1$. Subsequently it was reheated to $T=1$. The instantaneous configurational temperature (Eq. (10)) was computed once every 10 attempted moves per particle (that is, every $10 \mathrm{MC}$ steps) along with the configurational energy $E_{c}$ and pressure $P$. Results are shown in Fig. 1.

$T_{\text {config }}, E_{c}$, and $P$ show nearly immediate responses to the initial temperature quench. Because the quench is into the coexistence region, the system is initially far from equilibrium, and it takes a large number of Monte-Carlo moves for thermodynamic free energies to equilibrate. Figure 1 shows that $P$ and $E_{c}$ have not reached their asymptotic, equilibrium values even 5000 steps after the quench. Somewhat surprisingly, Figure 1 does show that $T_{\text {config }}$ equilibrates almost immediately and is nearly indistinguishable from the input temperature after only $\sim 200 \mathrm{MC}$ steps. In contrast to the pressure, $T_{\text {config }}$ relaxes monotonically after the temperature quenches. Upon reheating to $T=1$, we see a similarly rapid equilibration of $T_{\text {config }}$ relative to that of the pressure and energy. It is clear from this work that thermodynamic equilibrium is not a necessary condition for $T_{\text {config }}$ to be equal to the input temperature used in a Monte-Carlo program to generate a Markov 
chain.

When we compare the relative equilibration rates we find that those phase functions which depend on the highest derivatives of the potential relax fastest to their equilibrium values. Hence the order of relaxation rates is: $T_{\text {config }}$ is faster than $P$, which in turn equilibrates faster than $E_{c}$.

\subsection{Effect of simple coding errors}

To test the sensitivity of $T_{\text {config }}$ to programing or algorithm errors, the MC routine that is used to determine a trial move from a uniform distribution on the interval $[-1,1]$ was altered to return uniform random deviates on $[-0.5,1.5]$ instead. Attempted moves are thus biased toward the positive $\mathrm{x}$ and $\mathrm{y}$ directions, violating the rule of microscopic reversibility necessary to generate an irreducible Markov chain. ${ }^{3}$ Again, the simulation was performed on a 2D Lennard-Jones system, like that described in the previous section, with $N=50000$, at a reduced temperature of $T=1$. This time, $T_{\text {config }}$ did not agree with the input temperature but instead yielded a value of $0.9693 \pm 0.0002-$ not far from the input temperature but clearly statistically different from 1 . This particular programing error was thus easily detected from a computation of $T_{\text {config }}$.

Such an obvious mistake would probably not be encountered in practice, but other, more subtle errors that also violate microscopic reversibility may be encountered - particularly in complex MC computer programs. These are also the kind of errors that are perhaps the most difficult to detect. 


\subsection{Effect of system size}

Since terms of order $1 / N$ were neglected in the derivation of Eq. (10), ${ }^{4}$ the effect of system size on the behavior of $T_{\text {config }}$ was also studied. The same 2D MC simulation, at $T=1$, was performed, and the number of particles was varied from 50 to 100000 while the density was kept fixed. $T_{\text {config }}$ was computed from (10) once every $10 \mathrm{MC}$ steps and the values from many of these 'independent' configurations were averaged. The number of configurations was chosen so that the statistical uncertainty in the estimated temperatures $(\sim 0.02 \%)$ was independent of $N$. The result is shown in Fig. 2. Here, the uncertainties are smaller than the plotting symbols. For the particular state point chosen, it is clear that $T_{\text {config }}$ is always less than the input temperature. The discrepancy is about $8 \%$ for a system containing 50 particles, but less than $0.2 \%$ for one with 2000 particles. This is consistent with the omission of $1 / N$ terms from (10).

\subsection{Discontinuities in the potential}

In our derivation of the expression for $T_{\text {config }}$, we assumed that the potential function, and its first derivative with respect to the phase space coordinates, are continuous. When discontinuities are present, the derivation yielding Eq. (10) above is incorrect, and thus should not be used. This is obvious when one considers the case of a square-well potential. Here, a derivation that includes the effects on the configurational temperature when a particle crosses the square-well barrier would be needed. This is an important point since discontinuities are often present in simulations of systems with "continuous" potentials. Pair potentials are normally truncated and/or shifted to $u_{i j}\left(r_{\text {cut }}\right)=0$ at some cut-off distance to save computational resources. In Lennard-Jones systems, for example, this cutoff is commonly made at 2.5 particle diameters.

To demonstrate this, the effect that this cutoff distance has on $T_{\text {config }}$ was tested for the same Lennard-Jones system described above, except that the potential function was 
truncated at $r_{i j}=2.5$ and then shifted so that its value was $u_{i j}=0$ at $r_{i j}=2.5$ (a shift of approximately $1.6 \%$ of the well depth). The potential function is thus continuous, but its first derivative is not. In this test, $T_{\text {config }}$ was computed to be $0.9981 \pm 0.0004$ rather than the input temperature of $T=1$. This shows that $T_{\text {config }}$ is sensitive to even relatively minor errors or approximations in the simulation algorithm.

\section{CONCLUSION}

We have shown how a new expression for the temperature, based entirely on configurational rather than kinetic information, can be used to check that the temperature generated within a Monte Carlo simulation actually matches the specified input temperature. Although our derivation of the expression for the configurational temperature relies on thermodynamic equilibrium, thermodynamic equilibrium is not a necessary condition for the calculated configurational temperature to match the input temperature used to generate a Markov chain in Monte Carlo simulations. The configurational temperature relaxes rapidly. Checking the consistency between the value of the internal energy, and the first derivative of the potential energy (required for the estimation of the configurational temperature), provides a sensitive diagnostic for programing and algorithmic errors. This consistency is analogous to the energy conservation diagnostic employed for MD simulations except that diagnostic requires dynamic (rather than thermodynamic) consistency between the energy and the first derivative of the potential energy.

\section{Acknowledgement}

BDB thanks the staff at the Research School of Chemistry, Australian National University, for their hospitality during the visit where much of this work was performed. 


\section{References}

1. Rugh, H. H. (1997). "Dynamical approach to temperature", Phys. Rev. Lett. 78, 772; Rugh, H. H. (1997). “A geometric, dynamical approach to thermodynamics", http://xxx.lanl.gov/abs/chao-dyn/9703013.

2. G.A. Ayton, O.G. Jepps and D.J. Evans, (1998) “On the validity of Fourier's Law in systems with spatially varying strain rates", submitted to Mol. Phys.

3. See, for example Allen, M.P. and Tildesley, D.J. Computer Simulation of Liquids, Clarendon Press, Oxford, 1987, pp. 110-123.

4. We note here, that the expressions commonly used to calculate all thermodynamic properties, such as the pressure, free energy, etc., are also only accurate to first order in $N .^{3}$ 


\section{Figure Captions:}

Figure 1: (a) The configurational temperature, (b) pressure, and (c) configurational energy, for a system of particles quenched from $T=1$ to $T=0.1$ and then reheated (see text). Note that $T_{\text {config }}$ tracks the MC temperature rapidly even though $P$ and $E_{c}$ indicate that the system is still far from equilibrium.

Figure 2: The effect of system size on $T_{\text {config }}$ computed from Eq. (10). The temperature input to the MC simulation in this case was $T=1$. The difference between the computed $T_{\text {config }}$ and the input temperature is proportional to $1 / N$. 


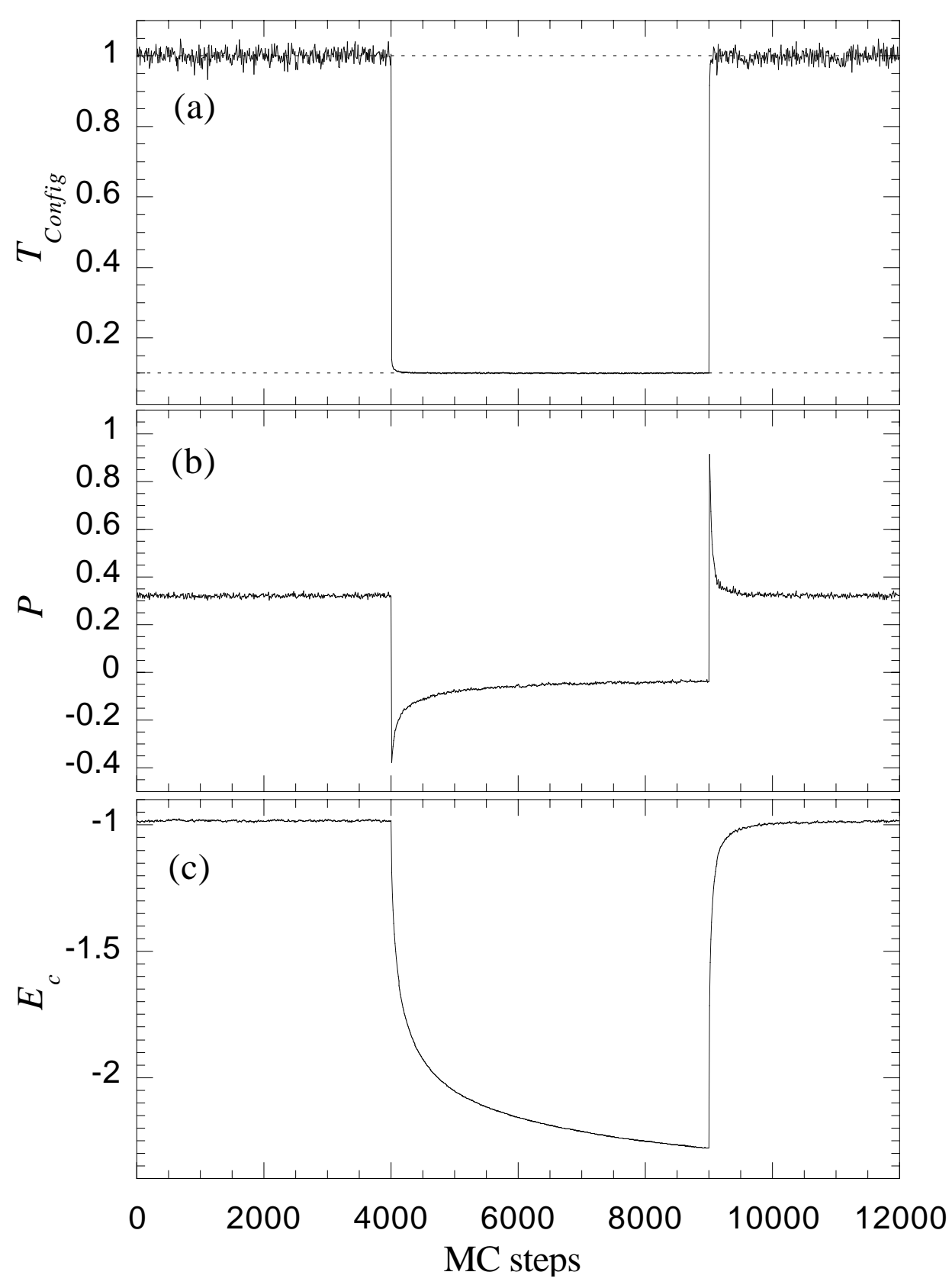

Figure 1: (a) The configurational temperature, (b) pressure , and (c) configurational energy, for a system of particles quenched from $T=1$ to $T=0.1$ and then reheated (see text). Note that $T_{\text {config }}$ tracks the MC temperature rapidly even though $P$ and $E_{c}$ indicate that the system is still far from equilibrium. 


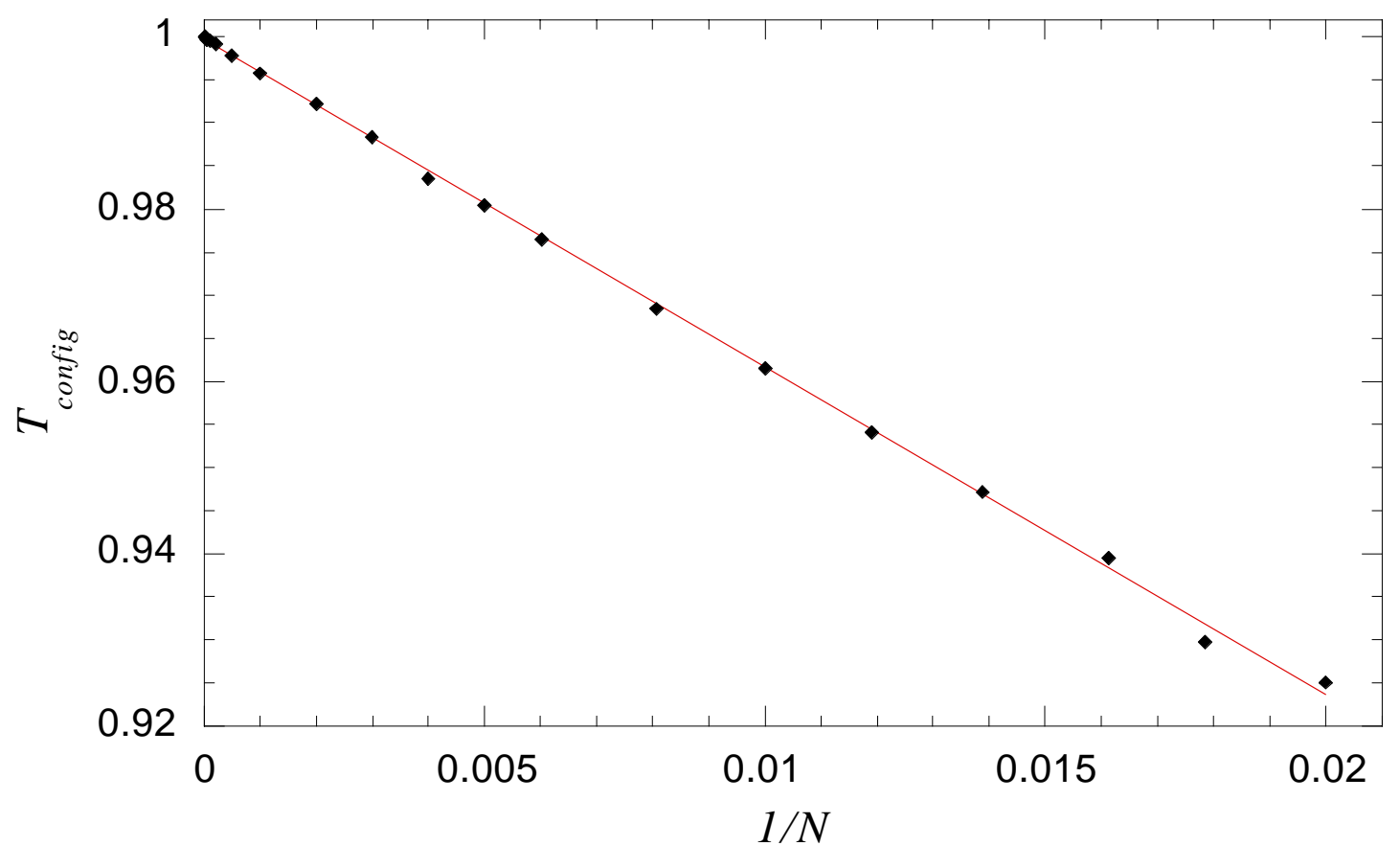

Figure 2: The effect of system size on $T_{\text {config }}$ computed from Eq. (10). The temperature input to the $\mathrm{MC}$ simulation in this case was $T=1$. The difference between the computed $T_{\text {config }}$ and the input temperature is proportional to $1 / N$. 